ARTICLE

\title{
Multispectral camouflage for infrared, visible, lasers and microwave with radiative cooling
}

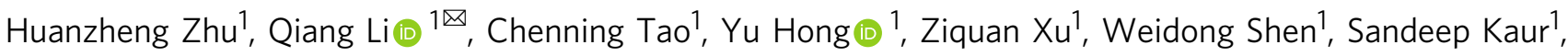 \\ Pintu Ghosh ${ }^{1} \&$ Min Qiu ${ }^{2,3 凶}$
}

Interminable surveillance and reconnaissance through various sophisticated multispectral detectors present threats to military equipment and manpower. However, a combination of detectors operating in different wavelength bands (from hundreds of nanometers to centimeters) and based on different principles raises challenges to the conventional single-band camouflage devices. In this paper, multispectral camouflage is demonstrated for the visible, mid-infrared (MIR, 3-5 and 8-14 $\mu \mathrm{m})$, lasers $(1.55$ and $10.6 \mu \mathrm{m})$ and microwave $(8-12 \mathrm{GHz})$ bands with simultaneous efficient radiative cooling in the non-atmospheric window (5-8 $\mu \mathrm{m})$. The device for multispectral camouflage consists of a $\mathrm{ZnS} / \mathrm{Ge}$ multilayer for wavelength selective emission and a CU-ITO-Cu metasurface for microwave absorption. In comparison with conventional broadband low emittance material $(\mathrm{Cr})$, the IR camouflage performance of this device manifests $8.4 / 5.9^{\circ} \mathrm{C}$ reduction of inner/surface temperature, and $53.4 / 13.0 \% \mathrm{IR}$ signal decrease in $\mathrm{mid} /$ long wavelength IR bands, at $2500 \mathrm{~W} \cdot \mathrm{m}^{-2}$ input power density. Furthermore, we reveal that the natural convection in the atmosphere can be enhanced by radiation in the non-atmospheric window, which increases the total cooling power from 136 $\mathrm{W} \cdot \mathrm{m}^{-2}$ to $252 \mathrm{~W} \cdot \mathrm{m}^{-2}$ at $150^{\circ} \mathrm{C}$ surface temperature. This work may introduce the opportunities for multispectral manipulation, infrared signal processing, thermal management, and energy-efficient applications.

\footnotetext{
${ }^{1}$ State Key Laboratory of Modern Optical Instrumentation, College of Optical Science and Engineering, Zhejiang University, Hangzhou, China. ${ }^{2}$ Key Laboratory of 3D Micro/Nano Fabrication and Characterization of Zhejiang Province, School of Engineering, Westlake University, Hangzhou, China. ${ }^{3}$ Institute of Advanced Technology, Westlake Institute for Advanced Study, Hangzhou, China. ${ }^{凶}$ email: qiangli@zju.edu.cn; qiumin@westlake.edu.cn
} 
$\mathrm{N}$ ature is the best inspiration for camouflage, which is extensively adopted by various species such as cephalopods, chameleons, and other animals to disguise themselves against predators ${ }^{1-4}$. In modern military and civil applications, camouflage technology aims to conceal the signature of objects and render them invisible from the potential threats ${ }^{5}$. Conventionally, camouflage is only confined in a particular spectral range, e.g., visible ${ }^{3,6}$, mid-infrared $(\mathrm{MIR})^{7-13}$, or microwave ${ }^{14}$. However, a combination of advanced detectors operating in different wavelength bands (e.g., visible to MWIR camera $^{15}$, SAR, and IR combination ${ }^{16}$ ) developed in recent years entails a compatible camouflage among different spectral bands, i.e., multispectral camouflage $e^{17-21}$.

Manipulating electromagnetic waves for multispectral camouflage is a challenging task due to different camouflage principles in different bands and the necessity to cover the wide spectrum range of wavelength from hundreds of nanometers (visible) to centimeters (microwave) ${ }^{15-17}$. To satisfy the requirements of multispectral camouflage, the spectral properties of the device should be: (i) low emittance in the MIR atmospheric windows i.e., mid-wavelength infrared (MWIR) 3-5 $\mu \mathrm{m}$ and long-wavelength infrared (LWIR) $8-14 \mu \mathrm{m}$ against thermal imagers and heat-seeking missiles ${ }^{19-26}$; (ii) characteristic reflection in the visible $(380-780 \mathrm{~nm})$ range for background matching 27,28 ; (iii) high absorbance in the microwave radar band (X-band, $8-12 \mathrm{GHz}$ ) for reducing radar cross-section (RCS) ${ }^{29}$ and (iv) high absorbance at the laser wavelengths $(1.55 \mu \mathrm{m}$ and $10.6 \mu \mathrm{m})^{30,31}$. According to the Stefan-Boltzmann law, the thermal radiation intensity is proportional to the fourth power of the surface temperature; therefore, reduction of the surface temperature is desired for IR camouflage ${ }^{32-35}$, especially for the military objectives with high surface temperature (e.g., converging nozzles of aircraft $(\sim 950 \mathrm{~K})^{36}$, tailpipe of aircraft $(\sim 740 \mathrm{~K})^{37}$, and funnels of naval ships $\left.(\sim 680 \mathrm{~K})^{38}\right)$. However, radiative heat transfer is blocked in traditional MIR camouflage with broadband low emittance, introducing heat instability ${ }^{39,40}$. In addition, camouflage against active detection systems including microwave and lidar further aggravates the heat instability due to absorbed microwave/laser energy ${ }^{18}$. To mitigate the severe heat instability and reduce surface temperature, high emittance is required in the MIR non-atmospheric window 5-8 $\mu \mathrm{m}$ for radiative cooling and wavelength-selective emitters have been proposed ${ }^{18,41-45}$. Although the MIR compatible multispectral camouflage including visible ${ }^{27,46,47}$, microwave ${ }^{18,48,49}$, or laser ${ }^{30,31}$ bands have been demonstrated, there is no existing material that simultaneously satisfies all the aforementioned requirements. Consequently, advanced technology for multispectral camouflage and radiative cooling still needs to be explored to ensure multispectral compatible functionality and effectiveness.

In this paper, we demonstrate multispectral camouflage for MIR, visible, laser, and microwave bands with efficient radiative cooling in the non-atmospheric window. By taking advantage of the wavelength difference of the spectral bands, the device realizes visible, MIR, and laser camouflage, as well as radiative cooling by the Ge/ZnS multilayer wavelength-selective emitter, and the microwave camouflage by the $\mathrm{Cu}$-ITO-Cu absorptive metasurface. This structure presents several advantageous characteristics: (1) low emittance of $0.11 / 0.12$ in the MWIR/LWIR bands for MIR camouflage, and high emittance of 0.61 in the nonatmospheric window for radiative cooling; (2) multiple visible colors for color matching in different backgrounds (e.g., soil, desert, water body, and vegetation); (3) high absorbance $(>0.9)$ in $\mathrm{X}$-band and (4) high absorbance at $1.55 \mu \mathrm{m}$ and $10.6 \mu \mathrm{m}$ lasers wavelengths. Compared with broadband low emittance material $\mathrm{Cr}$, the wavelength selective emitter shows a decrease of $8.4 / 5.9^{\circ} \mathrm{C}$ for inner/surface temperature and reduction of $53.4 / 13.0 \%$ for IR signal, with a heat source of $2500 \mathrm{~W} \cdot \mathrm{m}^{-2}$ power density. Furthermore, comparison experiments between vacuum and normal pressure reveal that the natural convection can be enhanced by radiation in the non-atmospheric window, and therefore the cooling power is enhanced to improve MIR camouflage performance.

\section{Results}

Multispectral camouflage. Multispectral camouflage depends on the detection principles in different wavelength bands, and the detectability of the wavelength spectrum (dashed boxes and short arrows in Fig. 1a) is closely related to the transmittance of electromagnetic waves of that wavelength through the atmosphere (light blue shaded area in Fig. 1a). In the MIR atmospheric window (blue dashed boxes), low emittance (blue arrow in Fig. 1b) is required as most thermal imagers and heat-seeking missiles operate in this wavelength region ${ }^{15}$, whereas in the nonatmospheric window (5-8 $\mu \mathrm{m}$, orange box in Fig. 1a), the thermal radiation intensity of an object with a moderate temperature, $\left(100-300^{\circ} \mathrm{C}\right)$ reaches the maximum, thus high emittance is required for radiative cooling without influencing MIR camouflage (orange arrow in Fig. 1b). At the typical lidar's laser wavelengths, e.g., $1.55 \mu \mathrm{m}$ and $10.6 \mu \mathrm{m}$ (purple short arrows in Fig. 1a), high absorbance is required for reducing the reflection or other back-scattering. On the other hand, high absorbance at $10.6 \mu \mathrm{m}$ is in conflict with the low emittance requirement in the LWIR band, hence requiring a sufficiently narrow bandwidth for a $10.6 \mu \mathrm{m}$ absorption peak (Fig. 1a). For the visible range $(380-780 \mathrm{~nm}$, colored dashed box in Fig. 1a), the reflectance spectrum is required to be close to the background to form the resembling color as the background. For the microwave range $(8-12 \mathrm{GHz}, \mathrm{X}$-band), high absorbance is demanded low reflectance or scattering (purple arrows in Fig. 1b), which is similar to the lidar.

To overcome the challenge of all spectral requirements with one structure, the combined structure is fabricated with different layers to satisfy corresponding spectral requirements. The spectral compatibility is ensured by leveraging the wavelength difference. The combined structure (Fig. 1b) consists of a Ge/ZnS multilayer wavelength-selective emitter (SE) for visible/lasers/MIR camouflage with radiative cooling and a $\mathrm{Cu}-\mathrm{ITO}-\mathrm{Cu}$ microwave absorptive metasurface.

For visible range, the spectrum is determined only by the thickness $\left(t_{\mathrm{ZnS}}\right.$ in Fig. $\left.2 \mathrm{c}\right)$ of the top $\mathrm{ZnS}$ layer as the beneath Ge layer $(0.72 \mu \mathrm{m})$ is opaque. By varying the $t_{\mathrm{ZnS}}$ from $30 \mathrm{~nm}$ to $270 \mathrm{~nm}$, anti-reflection peaks are formed at different wavelengths. Fig. 2a demonstrates the experimental reflectance for different $t_{\mathrm{ZnS}}$ and the corresponding colors with the optical image in the inset with CIE-1931 chromaticity coordinates $(x, y)$. The thickness of the top ZnS layer can be chosen to achieve background matching colors, e.g., $30 / 140 \mathrm{~nm}$ for soil/deserts background, $180 \mathrm{~nm}$ for clear water body background, and $220 \mathrm{~nm}$ for green vegetation background ${ }^{50}$.

For IR range, high reflectance (low emittance) in the MWIR and LWIR bands along with low reflectance (high emittance) in the non-atmospheric window is realized with all $\mathrm{ZnS} / \mathrm{Ge}$ multilayers. The thicknesses are optimized by the genetic algorithm, with the optimization targets of maximum absorbance in the non-atmospheric window, maximum absorbance at 10.6 $\mu \mathrm{m}$ and $1.55 \mu \mathrm{m}$ wavelengths, and minimum absorbance in both atmospheric windows. The measured band emittance at $100^{\circ} \mathrm{C}$ for MWIR/LWIR is $0.11 / 0.12$, and band emittance for nonatmospheric window $(5-8 \mu \mathrm{m})$ is 0.61 (see Supplementary note 1 for band emittance definition). The MIR spectra of the SE are hardly influenced by the top ZnS layer, due to its negligible 

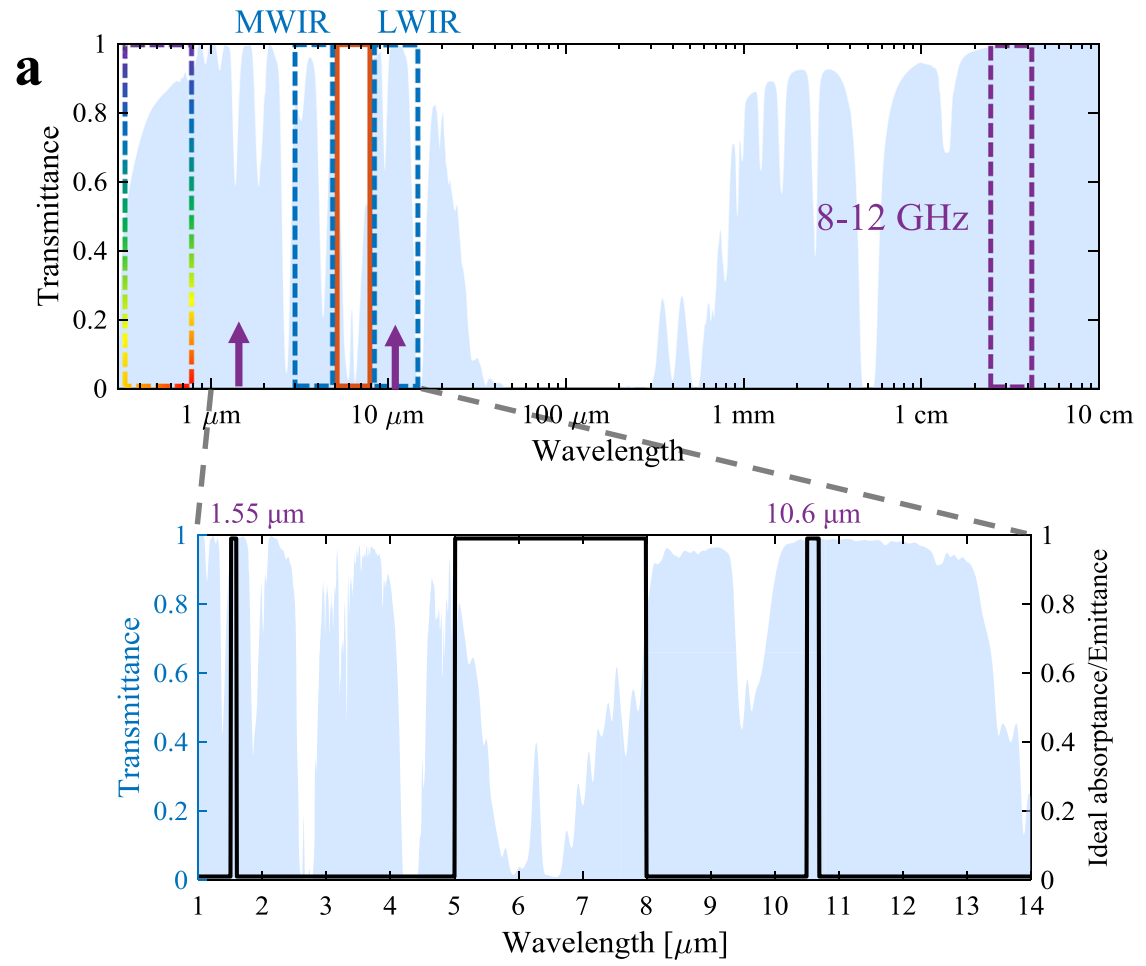

b Non-atmospheric window $(5-8 \mu \mathrm{m})$ : IR atmospheric window $(3-5,8-14 \mu \mathrm{m})$ :

High emittance

(Radiative cooling)

Visible (380-780 nm):

Spectral reflection

(color camouflage)
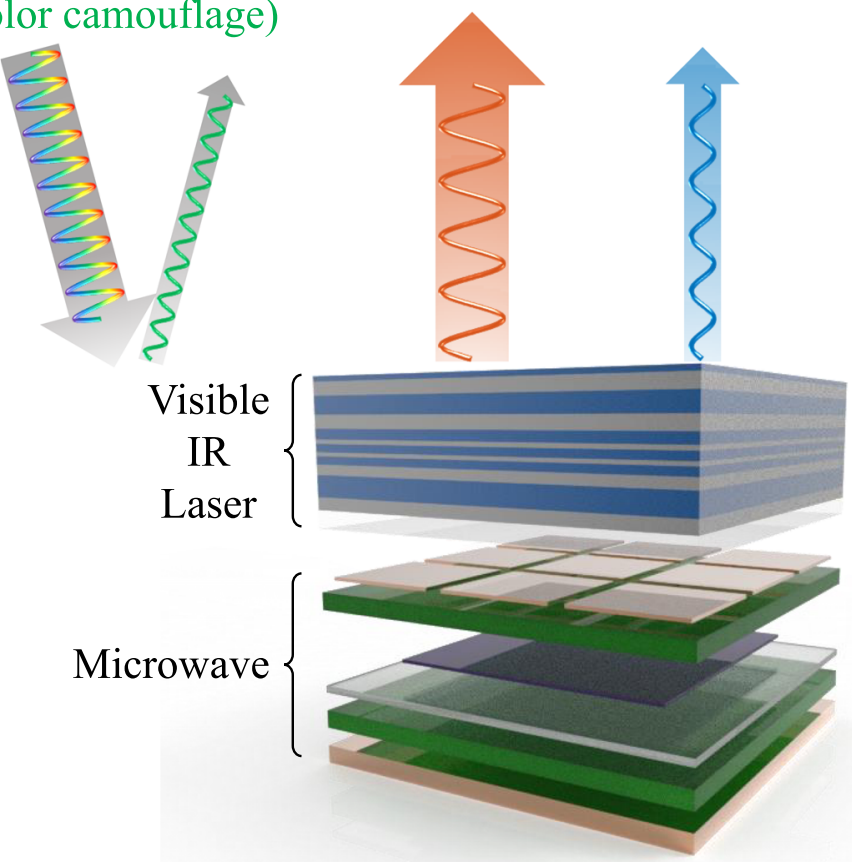

Low emittance

(IR camouflage)

Laser \& microwave radar:

\section{Low reflectance}

(radar camouflage)

Fig. 1 Atmosphere transmittance and scheme for multispectral camouflage. a Atmosphere transmittance spectrum for the electromagnetic wave (from visible to microwave) with detectable ranges indicated by dashed boxes and arrows. The IR part (1-14 $\mu \mathrm{m})$ is magnified and shown with the ideal absorbance/emittance spectrum. b Requirements of multispectral camouflage for each respective range, and the designed structure with ZnS/Ge multilayer selective emitter for visible, IR, and laser camouflage and Cu-ITO-Cu absorptive metasurface for microwave camouflage. 

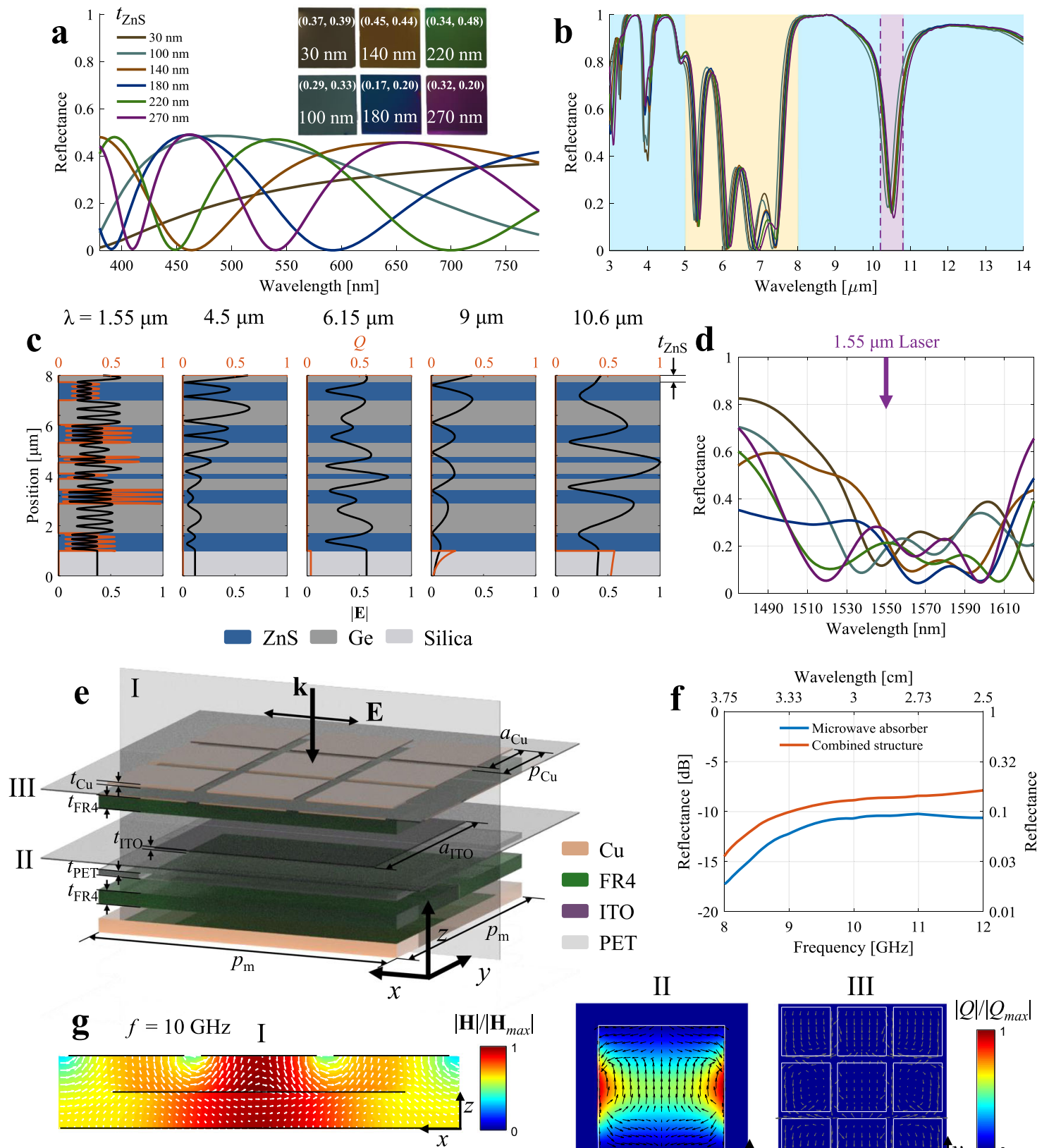

Arrow: E
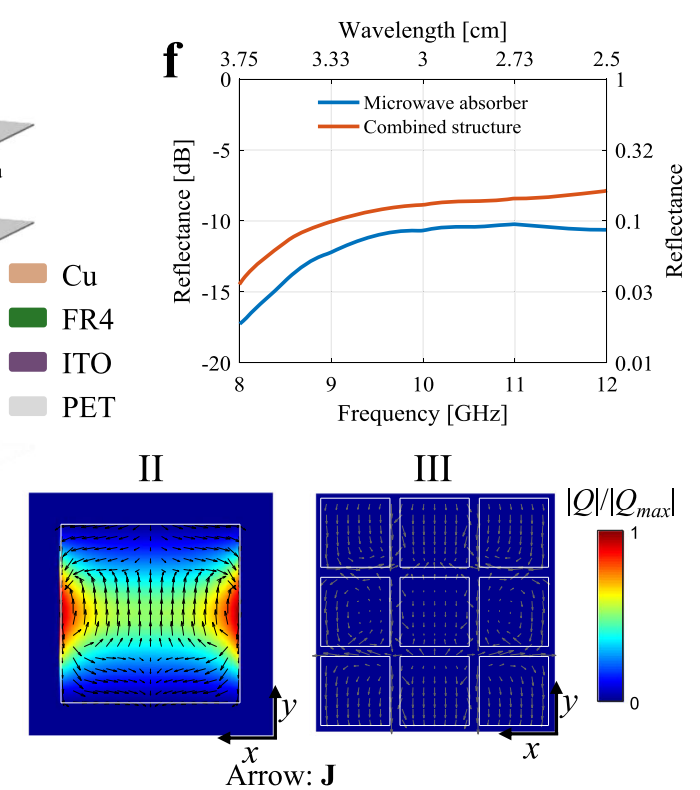

Fig. 2 Multispectral camouflage for visible, MIR, lasers, and microwave. a The measured visible reflectance and optical images for top ZnS layer of thickness varying from $30 \mathrm{~nm}$ to $270 \mathrm{~nm}$. The chromaticity coordinates $(x, y)$ are written on the corresponding optical images (insets). $\mathbf{b}$ The measured reflectance in the MIR range for the different values of top ZnS layer thickness. c The electric field $|\mathbf{E}|$ and resistive loss $Q$ in the $\mathrm{ZnS} / \mathrm{Ge}$ multilayer selective emitter for wavelengths of $1.55 \mu \mathrm{m}, 4.5 \mu \mathrm{m}, 6.15 \mu \mathrm{m}, 9 \mu \mathrm{m}$, and $10.6 \mu \mathrm{m}$. The thicknesses of the Ge/ZnS multilayer films are 0.721/0.982/0.721/0.559/ 0.234/0.438/0.206/0.438/0.552/1.18/0.701 $\mu \mathrm{m}$ (from top to bottom). d The reflectance spectrum near $1.55 \mu \mathrm{m}$. e The Cu-ITO-Cu microwave absorptive metasurface. $\mathbf{f}$ The reflectance spectra corresponding to the Cu-ITO-Cu microwave absorber metasurface and the combined structure. $\mathbf{g}$ The normalized magnetic field intensity (color) and electric field (arrow) on the cross-section I ( $x-z$ plane, parallel to polarization); the normalized resistive loss (color) and surface currents (arrow) on the cross-sections II and III, respectively ( $x-y$ plane, perpendicular to wave vector). The frequency is $10 \mathrm{GHz}$.

thickness compared to wavelength (Fig. 2b, see Section S3 for SE reflectance spectra for different $t_{\mathrm{ZnS}}$ ). Apart from the top $\mathrm{ZnS}$ layer, the thicknesses of the Ge/ZnS (gray/blue blocks) multilayer films from top to bottom are optimized as shown in Fig. $2 c$. The $\mathrm{Ge} / \mathrm{ZnS}$ layers form a one-dimensional photonic crystal with forbidden bands located at MWIR and LWIR ranges, as shown by the electric field $(|\mathbf{E}|)$ and resistive heat loss $(Q)$ distribution at $4.5 \mu \mathrm{m}$ and $9 \mu \mathrm{m}$ wavelengths (Fig. $2 \mathrm{c}$ ). For the non-atmospheric window outside the forbidden bands, the electric field does not decay within the Ge/ZnS layers, forming high absorbance within the lossy silica substrate.

For lidar's laser wavelength, the narrowband low reflectance at $10.6 \mu \mathrm{m}$ wavelength is realized with the fifth to seventh $\mathrm{Ge} / \mathrm{ZnS}$ layers (top to bottom, excluding the top $\mathrm{ZnS}$ layer), as shown by the electric field distribution at $10.6 \mu \mathrm{m}$ in the Fig. 2c. The forbidden band of the one-dimensional photonic crystal in LWIR is broken by these three layers, where high electric field intensity is formed. For another lidar's laser wavelength of $1.55 \mu \mathrm{m}$, the 
absorbance is mainly realized by the intrinsic loss within the $\mathrm{Ge}$ layers (extinction coefficient $k=0.0056$ at $1.55 \mu \mathrm{m}$ ). The measured reflectance value at $1.55 \mu \mathrm{m}$ wavelength for various $t_{\mathrm{ZnS}}$ is less than 0.3 , and therefore camouflage for $1.55 \mu \mathrm{m}$ lidar is achieved.

For the microwave X-band, low reflectance is observed for the $\mathrm{Cu}-\mathrm{ITO}-\mathrm{Cu}$ microwave absorber (the structure shown in Fig. 2e), which is characterized by a return loss lower than $-10 \mathrm{~dB}$, and the corresponding absorbance is higher than $90 \%$ (Fig. 2f). The periodic array of $\mathrm{Cu}$ square blocks with thickness $t_{\mathrm{Cu}}=0.18 \mu \mathrm{m}$, array period $p_{\mathrm{Cu}}=3.67 \mathrm{~mm}$, and block side length $a_{\mathrm{Cu}}=3.2 \mathrm{~mm}$ on flame retardant-4 (FR4, thickness $t_{\mathrm{FR} 4}=1 \mathrm{~mm}$ ) form a frequency selective surface for the $\mathrm{X}$-band. With the $\mathrm{Cu}$ substrate, the magnetic field is enhanced between the frequency selective surface and reflective substrate (Fig. $2 \mathrm{~g}$ ). With the electrically lossy ITO layer of thickness $t_{\mathrm{ITO}}=175 \mathrm{~nm}$, side length $a_{\mathrm{ITO}}=$ $8.1 \mathrm{~mm}$, and sheet resistance $35 \Omega \cdot \mathrm{sq}^{-1}$ on $175-\mu \mathrm{m}$-thick PET, the enhanced magnetic field, therefore, introduces high ohmic loss (Fig. 2g). Together with the selective emitter part, the return loss is still lower than $-8.25 \mathrm{~dB}$ for the combined structure, corresponding to the absorbance higher than $85 \%$. Therefore, the combined structure is compatible with MIR, visible, laser, and microwave camouflage, as well as radiative cooling.

IR camouflage demonstration. The camouflage for the MIR range is complicated due to spectral conflicts among MIR camouflage, radiative cooling, and laser camouflage. To demonstrate the performance of MIR camouflage, the SE part of the multispectral camouflage structure is compared with the $\mathrm{Cr}$ film on the same silica substrate as a conventional broadband low emittance surface, in terms of absolute temperature and radiative temperature reduction. For both SE and $\mathrm{Cr}$ cases, the constant power input is applied to the heater beneath the samples (Fig. 3a). The silica aerogel plate under the heater is used for thermal insulation, and the copper plate ensures horizontal temperature uniformity. The heater temperature $T_{h}$ and sample surface temperature $T_{s}$ are measured with the input power varying from $1.25 \mathrm{~W}$ to $25 \mathrm{~W}$ (Fig. 3b). Both the heater temperature $T_{h}$ and the surface temperature $T_{s}$ of the SE are lower than those of $\mathrm{Cr}$ for the same input power values, and the

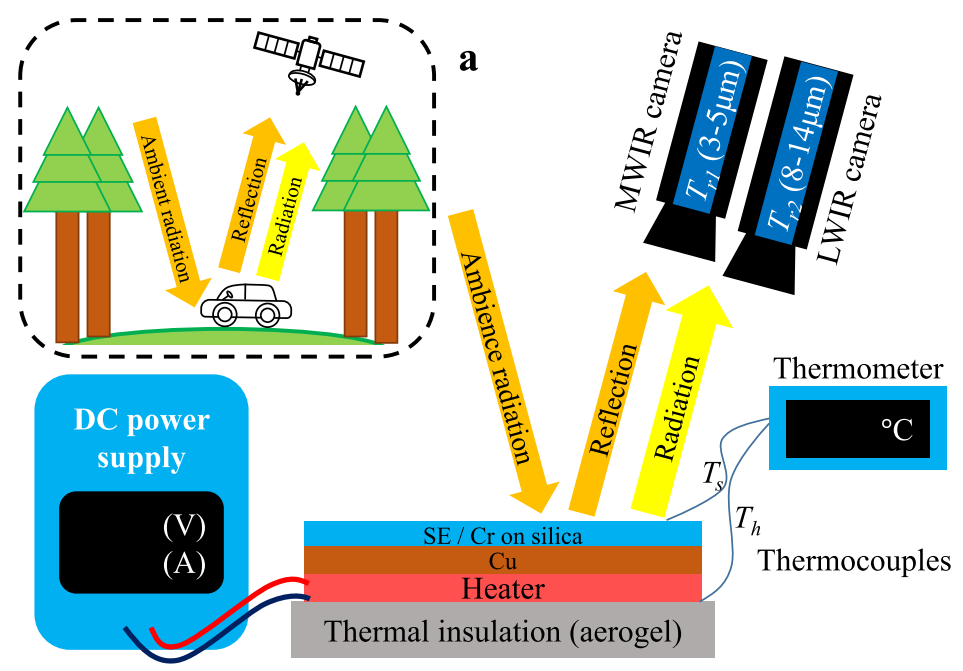

b
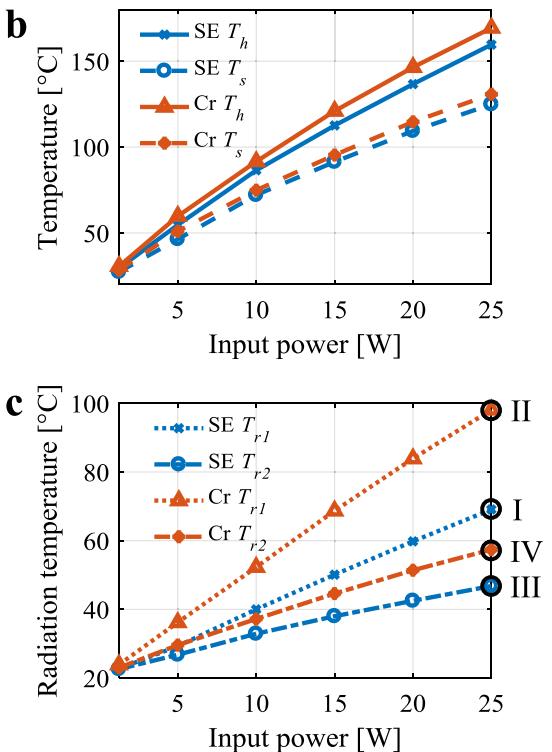

d $\quad$ MWIR $(3-5 \mu \mathrm{m})$

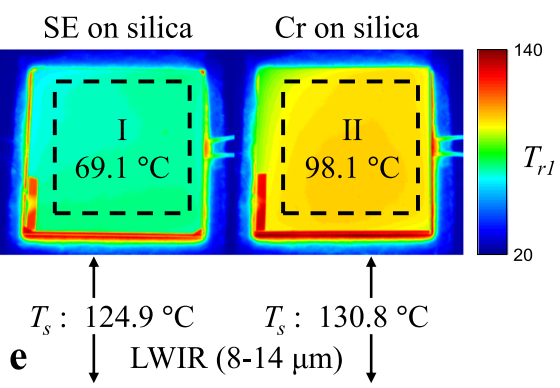

SE on silica $\quad$ Cr on silica

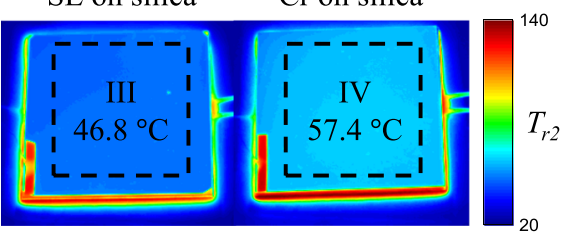

Fig. 3 IR camouflage demonstration. a Schematic diagram of the experimental configuration for IR camouflage and radiative cooling performance demonstration. b Measured heater/surface temperature $T_{h} / T_{s}$ for SE and $\mathrm{Cr}$ at different input heating power values. c Measured radiation temperature $T_{r 1} /$ $T_{r 2}$ in MWIR/LWIR band for SE and $\mathrm{Cr}$ at different input power values. d IR images of SE and Cr at 25 W input power captured by MWIR band thermal imager. e IR images of SE and $\mathrm{Cr}$ at $25 \mathrm{~W}$ captured by LWIR band thermal imager. 

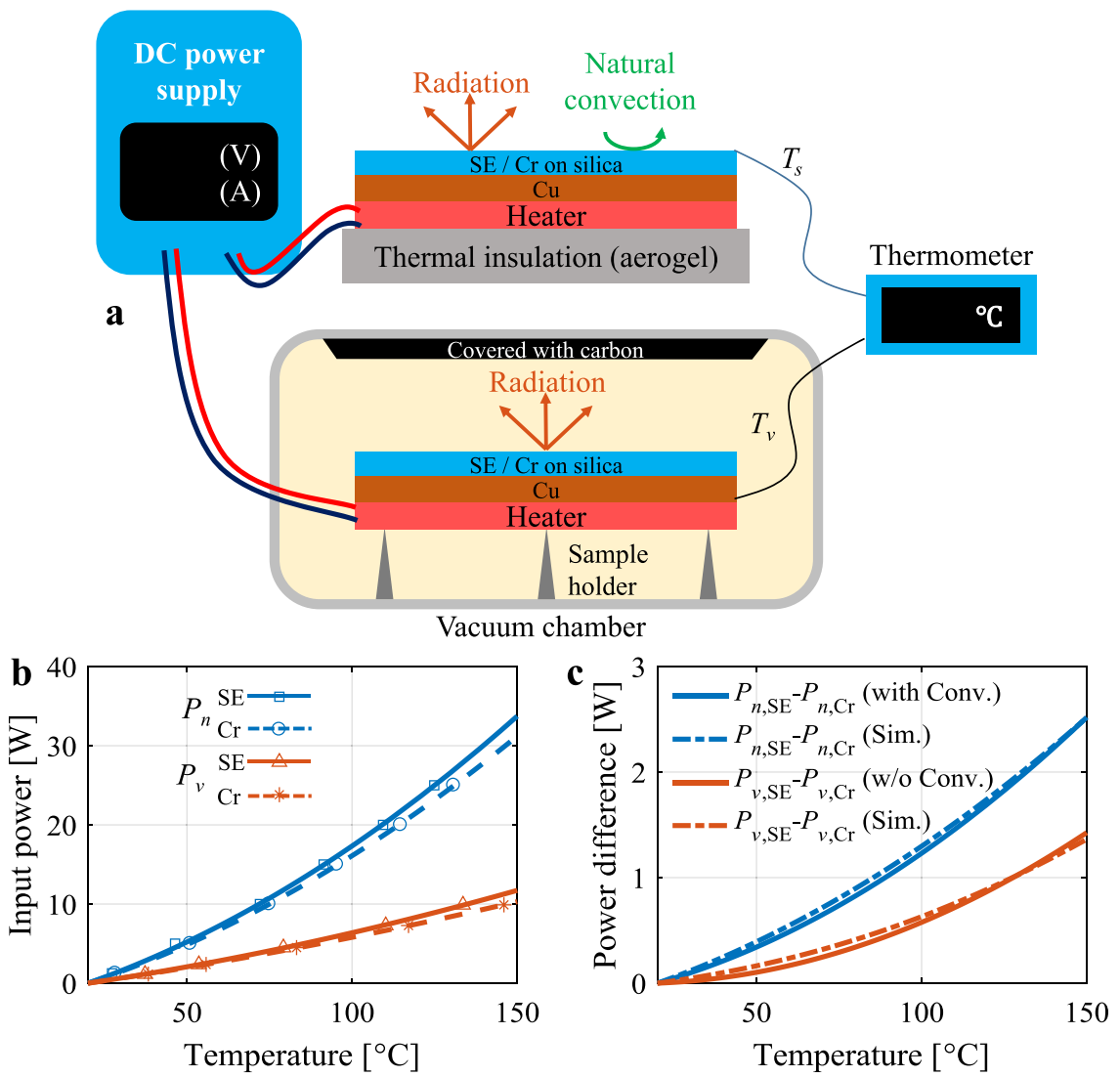

Fig. 4 Validation experiments of enhanced natural convection by radiation. a Schematic diagram showing a comparison of experimental setups between normal pressure and vacuum conditions. $\mathbf{b}$ The required input power for steady-state temperatures under normal pressure $\left(P_{n}\right)$ and vacuum $\left(P_{v}\right)$ conditions. c The input power difference between the $\mathrm{SE}$ and $\mathrm{Cr}$ at two conditions (with/without natural convection for normal pressure/vacuum) and simulated power differences with/without natural convection.

temperature difference between $\mathrm{SE}$ and $\mathrm{Cr}$ increases with the input power. At $25 \mathrm{~W}$ input power, $T_{h} / T_{s}$ of $\mathrm{SE}$ is about $8.4 / 5.9^{\circ} \mathrm{C}$ lower than that of $\mathrm{Cr}$, indicating efficient dissipation for internal/surface heat through radiative cooling.

The radiative (apparent) temperature $T_{r 1}$ and $T_{r 2}$ in MWIR and LWIR range, respectively, are measured for quantitatively evaluating the IR camouflage performance (Fig. 3c). Under the indoor configuration, both the reflected ambient radiation and radiation from the sample are collected by the thermal imagers, which is analogous to the scene of a forest or city with considerable ambient radiation shown in the dashed box in Fig. 3a. The radiative temperature of SE is significantly lower than $\mathrm{Cr}$, due to lower surface temperature $T_{s}$ and lower band emittance (see Supplementary note 9). At $25 \mathrm{~W}$ input, the surface temperatures $T_{s}$ are $124.9^{\circ} \mathrm{C}$ and $130.8^{\circ} \mathrm{C}$ for SE and $\mathrm{Cr}$, respectively. In the MWIR band, the radiation temperatures $T_{r 1}$ of $\mathrm{SE}$ (region I) and $\mathrm{Cr}$ (region II) are reduced to $69.1{ }^{\circ} \mathrm{C}$ and $98.1{ }^{\circ} \mathrm{C}$ (Fig. 3c), respectively, and in the LWIR band, the radiation temperature $T_{r 2}$ of SE and $\mathrm{Cr}$ are reduced to $46.8^{\circ} \mathrm{C}$ and $57.4^{\circ} \mathrm{C}$, respectively. Compared to $\mathrm{Cr}$, the SE shows a $53.4 \%(3.32 \mathrm{~dB})$ reduction of IR signal intensity in the MWIR range (band integrated radiation intensity for SE/Cr is $\left.22.6 / 48.6 \mathrm{~W} \cdot \mathrm{m}^{-2}\right)$ and a $13.0 \%(0.606 \mathrm{~dB})$ reduction of radiation intensity in LWIR range (band integrated radiation intensity for $\mathrm{SE} / \mathrm{Cr}$ is $229 / 264 \mathrm{~W} \cdot \mathrm{m}^{-2}$ ). The SE can endure a temperature of at least $300^{\circ} \mathrm{C}$ (see Supplementary note 10), which ensures its applicability for objects with high temperatures.

Enhanced natural convection by radiation. Under normal temperature and pressure, natural convection dominates heat transfer for stationary objects. Natural convection is detrimental in sub-ambient radiative cooling and should be avoided ${ }^{51-53}$; whereas for IR camouflage, as the radiative cooling operates at a temperature higher than ambiance for heat dissipation, the natural convection is beneficial to improve the cooling capacity by coupling to radiation. In the non-atmospheric window $(5-8 \mu \mathrm{m})$, the atmosphere is opaque mainly due to the absorption of the radiation power in the water vapor present in the ambient atmosphere; therefore, the radiation power is received by the water vapor in the ambient atmosphere. Enhancement of natural convection by radiation in the non-atmospheric window is demonstrated by the comparison experiments between the normal pressure condition and the vacuum condition (Fig. $4 \mathrm{a}$ ). In the normal pressure condition with a relative humidity of $60 \%$, both the natural convection and radiation are available for cooling of the sample under electric heating; whereas in the vacuum condition with a pressure of $\sim 1 \mathrm{~Pa}$, the natural convection is excluded. Other heat transfer channels, including conduction, side convection, and wire convection/radiation are regarded as parasitic loss, which are identical for $\mathrm{SE}$ and $\mathrm{Cr}$ at the same temperature.

The temperature is measured for different steady-state input power values for $\mathrm{SE}$ and $\mathrm{Cr}$ in both the normal pressure condition and the vacuum condition (Fig. 4b). To ensure the identity of radiative power between the normal pressure condition and the vacuum condition, the carbon surface is cooled with forced convection by air, and its temperature is close to ambient temperature, with the temperature monitoring for both sides (inside and outside the vacuum cavity). To reveal the influence of 
radiation on convection, the power differences between $\mathrm{SE}$ and $\mathrm{Cr}$ for both conditions are shown in Fig. 4c. If there is no coupling between radiation and convection, the power difference between $\mathrm{SE}$ and $\mathrm{Cr}$ should remain the same for both pressure conditions. Here, the power difference for the normal pressure condition (with convection) is larger than that of the vacuum condition (without convection), indicating enhanced natural convection due to coupled radiation. At the surface temperature of $150^{\circ} \mathrm{C}$, the power difference for normal pressure conditions is $2.52 \mathrm{~W}$, which is larger than that of vacuum conditions $(1.36 \mathrm{~W})$, corresponding to an increased power density difference to $252 \mathrm{~W} \cdot \mathrm{m}^{-2}$ from $136 \mathrm{~W} \cdot \mathrm{m}^{-2}$ (sample area of $0.01 \mathrm{~m}^{2}$ ). For both with/without convection, the experimental power differences (solid lines) are consistent with the simulations (dashdotted lines, see Supplementary note 9). The natural convection in the atmosphere can be enhanced with radiation in the nonatmospheric window, which provides a new approach for increasing the cooling power of devices when forced convection and radiative cooling in the atmospheric window are not available. This is especially significant for IR camouflage in which the radiation in the atmospheric window is strictly forbidden.

\section{Discussion}

Multispectral camouflage for visible, IR, laser, and microwave ranges is demonstrated together with efficient radiative cooling through the multilayer wavelength-selective emitter and the microwave metasurface. First, multispectral camouflage is realized with the design based on wavelength difference among MIR, visible, laser, and microwave ranges, whose spectral compatibility is ensured by the layer-wise functionality. Second, the utilization of SE not only resolves the spectral conflict between IR camouflage and radiative cooling, as well as laser camouflage but also enhances the performance of IR camouflage due to excellent radiative cooling. Third, it is revealed that the radiation in the non-atmospheric window can further enhance the natural convection and therefore increase the cooling power, which is significant in applications when other heat transfer channels are restricted. Last, the SE based on $\mathrm{ZnS} / \mathrm{Ge}$ multilayer films and the microwave metasurface based on common PCB processes are easy to fabricate and therefore hold potential for large-area applications. Ultimately, this work may open the opportunities for simultaneous manipulation of multispectral electromagnetic waves and heat transfer, thus paving the way towards military and civil applications, thermal management, and energy-efficient devices $^{54-61}$.

\section{Methods}

Selective emitter fabrication. The $\mathrm{ZnS} / \mathrm{Ge}$ multilayer film was deposited using the E-beam evaporation technique on a silica substrate with deposition rates of $1.5 \mathrm{~nm} / \mathrm{s}$ $(\mathrm{ZnS})$ and $0.5 \mathrm{~nm} / \mathrm{s}(\mathrm{Ge})$.

Optical characterization. The reflectance spectrum in the visible/NIR band was measured by a spectrophotometer (Cary7000, Agilent). The reflectance in the MIR band was measured by the FTIR microscope (Hyperion 1000, Brucker) with an FTIR spectrometer (Vertex 70, Brucker) through an MCT detector. The reflectance in the microwave band was measured by a vector network analyzer $(8722 \mathrm{C}$, Keysight) with a sample size of $200 \mathrm{~mm} \times 200 \mathrm{~mm}$.

Radiation/absolute temperature measurements. The radiation temperature was measured with an LWIR camera (Blackbird precision IR-OEM-Module, Jenoptik) with a detection wavelength range of 8-14 $\mu \mathrm{m}$ and an MWIR camera (FAST M200, Telops) with a detection wavelength range of $3-5 \mu \mathrm{m}$. The absolute temperature was measured by thermocouples (5TC-TT-K-30-36, Omega) at normal pressure and thermal resistors (Pt100, TZ-CMEI) in the vacuum with a thermometer (THTZ408R, Tenghui, Ningbo).
Simulations. The heat transfer simulation was performed with a heat transfer module and surface-to-surface radiation module in COMSOL Multiphysics. The microwave metasurface was designed with a radio frequency module (electromagnetic waves, frequency domain) in COMSOL Multiphysics. The electric field distribution is also simulated with a radio frequency module (electromagnetic waves, frequency domain) in COMSOL Multiphysics (the relative permittivity of $\mathrm{Ge} / \mathrm{ZnS}$ are available in Supplementary note 2).

\section{Data availability}

The data that support the plots within this paper and other findings of this study are available from the corresponding author upon reasonable request.

\section{Code availability}

Codes used for this work are available from the corresponding author upon reasonable request.

Received: 8 July 2020; Accepted: 23 February 2021; Published online: 22 March 2021

\section{References}

1. Xu, C., Stiubianu, G. T. \& Gorodetsky, A. A. Adaptive infrared-reflecting systems inspired by cephalopods. Science 359, 1495-1500 (2018).

2. Phan, L. et al. Reconfigurable infrared camouflage coatings from a cephalopod protein. Adv. Mater. 25, 5621-5625 (2013).

3. Teyssier, J., Saenko, S. V., Marel, Dvander \& Milinkovitch, M. C. Photonic crystals cause active colour change in chameleons. Nat. Commun. 6, 6368 (2015).

4. Zhang, X., Zhang, Z. G., Wang, Q., Zhu, S. N. \& Liu, H. Controlling thermal emission by parity-symmetric fano resonance of optical absorbers in metasurfaces. ACS Photon. 6, 5-10 (2019).

5. Xie, X. et al. Plasmonic metasurfaces for simultaneous thermal infrared invisibility and holographic illusion. Adv. Funct. Mater. 28, 1706673 (2018).

6. Pikul, J. H. et al. Stretchable surfaces with programmable 3D texture morphing for synthetic camouflaging skins. Science 358, 210-214 (2017).

7. Xiao, L. et al. Fast Adaptive thermal camouflage based on flexible VO2/ Graphene/CNT Thin Films. Nano Lett. 15, 8365-8370 (2015).

8. Salihoglu, O. et al. Graphene-based adaptive thermal camouflage. Nano Lett. 18, 4541-4548 (2018).

9. Li, Y., Bai, X., Yang, T., Luo, H. \& Qiu, C. W. Structured thermal surface for radiative camouflage. Nat. Commun. 9, 273 (2018).

10. Qu, Y. et al. Thermal camouflage based on the phase-changing material GST. Light Sci. Appl. 7, 26 (2018).

11. Peng, Y. G., Li, Y., Cao, P. C., Zhu, X. F. \& Qiu, C. W. 3D printed meta-helmet for wide-angle thermal camouflages. Adv. Funct. Mater. 30, 2002061 (2020).

12. Liu, X. \& Padilla, W. J. Thermochromic infrared metamaterials. Adv. Mater. 28, 871-875 (2016).

13. Lyu, J. et al. Nanofibrous Kevlar aerogel films and their phase-change composites for highly efficient infrared stealth. ACS Nano 13, 2236-2245 (2019).

14. Bai, B., Li, X., Xu, J. \& Liu, Y. Reflections of electromagnetic waves obliquely incident on a multilayer stealth structure with plasma and radar absorbing material. IEEE Trans. Plasma Sci. 43, 2588-2597 (2015).

15. Gerken, M., Fritze, J., Münzberg, M. \& Weispfenning, M. Military reconnaissance platform for the spectral range from the visible to the MWIR. Infrared Technol. Appl. XLIII 10177, 101770 (2017).

16. Kim, S., Song, W. J. \& Kim, S. H. Robust ground target detection by SAR and IR sensor fusion using adaboost-based feature selection. Sensors 16, 1117 (2016).

17. Kumar, N. \& Dixit, A. Nanotechnology for Defence Applications. (Springer International Publishing, 2019).

18. Kim, T., Bae, J. Y., Lee, N. \& Cho, H. H. Hierarchical metamaterials for multispectral camouflage of infrared and microwaves. Adv. Funct. Mater. 29, 1807319 (2019).

19. Wang, Z., Cheng, Y., Nie, Y., Wang, X. \& Gong, R. Design and realization of one-dimensional double hetero-structure photonic crystals for infrared-radar stealth-compatible materials applications. J. Appl. Phys. 116, 054905 (2014).

20. Zhang, W., Xu, G., Zhang, J., Wang, H. \& Hou, H. Infrared spectrally selective low emissivity from Ge/ZnS one-dimensional heterostructure photonic crystal. Opt. Mater. 37, 343-346 (2014).

21. Miao, L. et al. Heterogeneous doped one-dimensional photonic crystal with low emissivity in infrared atmospheric window. Opt. Eng. 55, 057101 (2016).

22. Chandra, S., Franklin, D., Cozart, J., Safaei, A. \& Chanda, D. Adaptive multispectral infrared camouflage. ACS Photon. 5, 4513-4519 (2018). 
23. $\mathrm{Xu}, \mathrm{Z}$. et al. Spatially resolved dynamically reconfigurable multilevel control of thermal emission. Laser Photon. Rev. 14, 1-9 (2020).

24. Xu, L., Dai, G. \& Huang, J. Transformation multithermotics: controlling radiation and conduction simultaneously. Phys. Rev. Appl. 13, 1 (2020).

25. Shahsafi, A. et al. Temperature-independent thermal radiation. Proc. Natl Acad. Sci. USA 116, 26402-26406 (2019).

26. Inoue, T., Zoysa, M., De, Asano, T. \& Noda, S. Realization of dynamic thermal emission control. Nat. Mater. 13, 928-931 (2014).

27. Qi, D. et al. Effective strategy for visible-infrared compatible camouflage: surface graphical one-dimensional photonic crystal. Opt. Lett. 43, 5323 (2018).

28. Morin, S. A. et al. Camouflage and display for soft machines. Science 337, 828-832 (2012).

29. Zhang, C. et al. Flexible and transparent microwave-infrared bistealth Structure. Adv. Mater. Technol. 4, 4-11 (2019).

30. Chen, X., Tian, C., Che, Z. \& Chen, T. Selective metamaterial perfect absorber for infrared and $1.54 \mu \mathrm{m}$ laser compatible stealth technology. Optik 172, 840-846 (2018).

31. Zhang, J. K., Shi, J. M., Zhao, D. P., Wang, Q. C. \& Wang, C. M. Realization of compatible stealth material for infrared, laser and radar based on onedimensional doping-structure photonic crystals. Infrared Phys. Technol. 85, 62-65 (2017).

32. Zhu, H. et al. High-temperature infrared camouflage with efficient thermal management. Light Sci. Appl. 9, 60 (2020).

33. Luo, H. et al. An ultra-thin colored textile with simultaneous solar and passive heating abilities. Nano Energy 65, 103998 (2019).

34. Xu, L. \& Huang, J. Metamaterials for manipulating thermal radiation: transparency, cloak, and expander. Phys. Rev. Appl. 12, 044048 (2019).

35. Park, J. H., Han, S. E., Nagpal, P. \& Norris, D. J. Observation of thermal beaming from tungsten and molybdenum bull's eyes. ACS Photon. 3, 494-500 (2016).

36. Baranwal, N. \& Mahulikar, S. P. Infrared signature of aircraft engine with choked converging nozzle. J. Thermophys. Heat. Tr. 30, 854-862 (2016).

37. Mahulikar, S. P., Rao, G. A. \& Kolhe, P. S. Infrared signatures of low-flying aircraft and their rear fuselage skin's emissivity optimization. J. Aircr. 43, 226-232 (2006).

38. Thompson, J., Vaitekunas, D. \& Birk, A. M. IR signature suppression of modern naval ships. In Proceedings of ASNE 21st Century Combatant Technology Symposium vol. 1, pp. 27-30 (ASNE, Virginia, USA, 1998).

39. Du, K. et al. Control over emissivity of zero-static-power thermal emitters based on phase changing material GST. Light Sci. Appl. 6, 16194 (2017).

40. Shi, N. N. et al. Keeping cool: Enhanced optical reflection and radiative heat dissipation in Saharan silver ants. Science 349, 298-301 (2015).

41. Asano, T. et al. Near-infrare-to-visible highly selective thermal emitters based on an intrinsic semiconductor. Sci. Adv. 2, e1600499 (2016).

42. Dyachenko, P. N. et al. Controlling thermal emission with refractory epsilonnear-zero metamaterials via topological transitions. Nat. Commun. 7, 11809 (2016).

43. Zhang, X., Liu, H., Zhang, Z. G., Wang, Q. \& Zhu, S. N. Controlling thermal emission of phonon by magnetic metasurfaces. Sci. Rep. 7, 1-8 (2017).

44. Liu, X. \& Padilla, W. J. Reconfigurable room temperature metamaterial infrared emitter. Optica 4, 430 (2017).

45. Baranov, D. G. et al. Nanophotonic engineering of far-field thermal emitters. Nat. Mater. 18, 920-930 (2019).

46. Pan, M. et al. Multi-band middle-infrared-compatible camouflage with thermal management via simple photonic structures. Nano Energy 69, 104449 (2019).

47. Li, M., Liu, D., Cheng, H., Peng, L. \& Zu, M. Manipulating metals for adaptive thermal camouflage. Sci. Adv. 6, eaba3494 (2020).

48. Xu, C. et al. Hybrid metasurfaces for infrared-multiband radar stealthcompatible materials applications. IEEE Access 7, 147586-147595 (2019).

49. Zhang, C. et al. An ultralight and thin metasurface for radar-infrared bistealth applications. J. Phys. D. Appl. Phys. 50, 444002 (2017).

50. Shaw, G. A. \& Burke, H. K. Spectral imaging for remote sensing. Linc. Lab. J. 14, 3-28 (2003).

51. Goldstein, E. A., Raman, A. P. \& Fan, S. Sub-ambient non-evaporative fluid cooling with the sky. Nat. Energy 2, 1-7 (2017).
52. Raman, A. P., Anoma, M. A., Zhu, L., Rephaeli, E. \& Fan, S. Passive radiative cooling below ambient air temperature under direct sunlight. Nature $\mathbf{5 1 5}$ 540-544 (2014)

53. Hsu, P. et al. Radiative human body cooling by nanoporous polyethylene textile. Science 353, 1019-1024 (2016).

54. Hong, S., Shin, S. \& Chen, R. An adaptive and wearable thermal camouflage device. Adv. Funct. Mater. 1909788, 1-7 (2020).

55. Sakat, E. et al. Enhancing thermal radiation with nanoantennas to create infrared sources with high modulation rates. Optica 5, 175 (2018).

56. Ma, Q. \& Cui, T. J. Information metamaterials: bridging the physical world and digital world. PhotoniX 1, 1-32 (2020).

57. Shrestha, S. et al. Indium tin oxide broadband metasurface absorber. ACS Photon. 5, 3526-3533 (2018).

58. Peng, L., Liu, D., Cheng, H., Zhou, S. \& Zu, M. A multilayer film based selective thermal emitter for infrared stealth technology. Adv. Opt. Mater. 6 1801006 (2018)

59. Zhai, Y. et al. Scalable-manufactured randomized glass-polymer hybrid metamaterial for daytime radiative cooling. Science 355, 1062-1066 (2017).

60. Zhou, L. et al. A polydimethylsiloxane-coated metal structure for all-day radiative cooling. Nat. Sustain. 2, 718-724 (2019).

61. Mandal, J. et al. Hierarchically porous polymer coatings for highly efficient passive daytime radiative cooling. Science 362, 315-319 (2018).

\section{Acknowledgements}

This work was supported by the National Key Research and Development Program of China (2017YFA0205700 and 2017YFE0100200) and the National Natural Science Foundation of China (Grant Nos. 61975181, 61775194, and 61950410608).

\section{Author contributions}

Q.L. conceived the idea and supervised the project together with M.Q. H.Z. and C.T. performed the experiments. H.Z. and Z.X. performed the calculations. H.Z., Y.H., and W.S. fabricated the devices. Q.L., H.Z., and M.Q. analyzed the data. Q.L., H.Z., S.K., and P.G. wrote the manuscript. All authors contributed to the final version of the manuscript.

\section{Competing interests}

The authors declare no competing interests.

\section{Additional information}

Supplementary information The online version contains supplementary material available at https://doi.org/10.1038/s41467-021-22051-0.

Correspondence and requests for materials should be addressed to Q.L. or M.Q.

Reprints and permission information is available at http://www.nature.com/reprints

Publisher's note Springer Nature remains neutral with regard to jurisdictional claims in published maps and institutional affiliations.

Open Access This article is licensed under a Creative Commons Attribution 4.0 International License, which permits use, sharing, adaptation, distribution and reproduction in any medium or format, as long as you give appropriate credit to the original author(s) and the source, provide a link to the Creative Commons license, and indicate if changes were made. The images or other third party material in this article are included in the article's Creative Commons license, unless indicated otherwise in a credit line to the material. If material is not included in the article's Creative Commons license and your intended use is not permitted by statutory regulation or exceeds the permitted use, you will need to obtain permission directly from the copyright holder. To view a copy of this license, visit http://creativecommons.org/ licenses/by/4.0/.

(C) The Author(s) 2021 